Review Article

\title{
Identifying Physicians' and Nurses' Nutrition Knowledge Using Validated Instruments: A Systematic Narrative Review
}

\author{
Jamie Zeldman, Jeanette Mary Andrade* \\ Food Science and Human Nutrition Department, University of Florida, Gainesville, USA \\ Email address: \\ jzeldman@ufl.edu (J.Zeldman), jandrade1@ufl.edu (J. M. Andrade) \\ ${ }^{*}$ Corresponding author
}

\section{To cite this article:}

Jamie Zeldman, Jeanette Mary Andrade. Identifying Physicians' and Nurses' Nutrition Knowledge Using Validated Instruments: A Systematic Narrative Review. International Journal of Nutrition and Food Sciences. Vol. 9, No. 2, 2020, pp. 43-53. doi: 10.11648/j.ijnfs.20200902.12

Received: March 10, 2020; Accepted: March 26, 2020; Published: April 13, 2020

\begin{abstract}
Physicians and nurses, who are knowledgeable in nutrition, improve patients' health outcomes. However, limited information is provided about the areas of nutrition they are knowledgeable in. This review sought to identify physicians' and nurses' nutrition knowledge through validated instruments. A systematic narrative review of the literature was conducted. Three databases - PubMed Central, Science Direct and Embase databases were searched from 1990 until December 2019. Retrieved studies were screened through a predetermined inclusion criterion and data extraction of included studies occurred. Quality assessment and risk of bias of included articles was completed. Thirty-three articles met the inclusion criteria. Instruments to identify nutrition knowledge varied among each study. Mean percentages of nutrition knowledge were between $32.5 \%$ correct to $72 \%$ correct. Nutrition knowledge was highest in the areas of nutrients' roles, and food sources/macronutrients, whereas knowledge was lowest in the area of providing medical nutrition therapy. In general, physicians and nurses who were older, considered a specialist, held an advanced degree and/or had more years of practice had higher nutrition knowledge scores. Overall, literature about physicians' and nurses' nutrition knowledge is heterogeneous and scant as well as the instruments used to measure this knowledge. Within these limits, nutrition knowledge may be improved in certain areas.
\end{abstract}

Keywords: Nutrition Knowledge, Validated Instruments, Physicians, Nurses

\section{Introduction}

In 2016, globally, $71 \%$ of deaths were attributed to non-communicable diseases (NCDs) - cardiovascular disease, cancer, diabetes, and chronic lung diseases [1]. Even though there are several modifiable risk factors that contributes to NCDs, one that is identified the most is a poor nutritious diet (e.g. low consumption of fruits, vegetables, whole grains and high intake of processed meats, refined sugars, salt) [2-4]. The combination of inadequate nutrition interventions and poor nutritional management contribute to the development and progression of these NCDs, which often leads to longer hospital stays and subsequent increases in healthcare costs [5]. For instance, among the Canadian adult population, the risk of cardiovascular disease was reduced by $4 \%$ and total premature death rate was reduced by $5 \%$ with adults consuming at least five servings of fruits and vegetables daily [6]. Furthermore, it was estimated that more than 30,000 deaths could have been delayed or avoided if the population consumed a diet that aligned with the dietary guidelines [7]. One approach that may help individuals consume a nutritious diet is physicians and nurses educating their patients about nutrition.

Patients prefer physicians to inform them about nutrition and the relation of their diet to the prevention and treatment of diseases [8-11]. However, insufficient nutrition knowledge has been one of the main barriers to providing adequate, high quality nutritional care, to their patients [1215]. After a 1989 report showed a lack of nutrition curriculum in medical schools, the 1990 National Nutrition Monitoring and Research Act empowered medical schools to 
include nutrition in the curriculum [16]. Nonetheless, more than half of graduating medical students [17] and nurses [18, 19] reported that the time dedicated to nutrition education is inadequate. Furthermore, the nutrition care practices of physicians and nurses is strongly influenced by their nutrition knowledge [20]. Nutrition knowledge, though, is a concept that may be interpreted in various ways.

Nutrition knowledge is defined as the ability to identify basic facts about food and nutrients and the effect on one's body [21, 22]. Nutrition knowledge is determined by a minimum of two of the following concepts: food groups, balanced diets, current dietary guidelines, sources of nutrients, storage and preparation of food, use of food labels, and the relationship between nutrition and disease [21-24]. For physicians and nurses, these general concepts are generally discussed in their medical and nursing schools, yet the time devoted to these topics are limited and may only be presented once in their two- or four-year curriculum [18, 2527]. For at least physicians, they may be introduced to specific nutrition information such as biochemistry (e.g. carbohydrate metabolism) and vitamin deficiency states (e.g. iron-deficiency anemia). Although, there lacks more formal nutrition education that integrates a biochemical issue or deficiency to nutrients or a diet to improve their nutritional status [25]. Even though physicians and nurses may not perceive they receive adequate nutrition education in a formal institution, they may acquire nutrition knowledge through other mechanisms such as continuing education, conferences, or independent reading of scientific nutrition literature [28-32]. Other factors that may contribute to nutrition knowledge is years of practice in their respective field and age [30]. Regardless of the method to which a physician and nurse has been exposed to nutrition information, it is critical to assess that knowledge, through validated instruments.

Validating an instrument determines the degree the instrument measures what it is supposed to measure. The validation process is complex and time intensive, however, to ensure quality of data from an instrument, it is necessary. There are three main methods to validate an instrument content, criterion-related, and construct. An instrument that has been deemed validated should contain the following components: simplicity and viability, reliability and precision in the words, adequate for the problem intended to measure, reflects an underlying theory or concept to be measured, and is capable of measuring change [33-35]. Even though an instrument may have undergone rigorous validation for a specific population, that instrument may only be valid for that population. 34 For example, a nutrition knowledge questionnaire has been validated for health professionals in the United States. If this instrument was to be used for health professionals within Central America, it must undergo another validation process to ensure it can be used on that population. Several nutrition knowledge instruments currently exist to determine nutrition knowledge among physicians and nurses. Although, the instruments used might not have been validated, thus the results have to be interpreted with caution. Therefore, the purpose of this systematic narrative review was to identify physicians' and nurses' nutrition knowledge through validated instruments.

\section{Methods}

This systematic review was conducted following the Preferred Reporting Items for Systematic Review and Meta-analysis (PRISMA) [36]. Neither humans nor animals were involved in this study, therefore no IRB approval was acquired.

\subsection{Search Strategy}

By guidance from the protocol of Cochrane [37], one researcher (J. Z.) utilized three databases: PubMed Central, Science Direct and Embase to find eligible articles between January 11990 until December 15 2019. In the Medical Subject Headings (MeSH) a combination of using 'or' and 'and' were used to search for the articles. Selection of the articles had to include nutrition knowledge, validated instruments, and physicians/nurses, as indicated in table 1.

According to Bramer and colleagues [39], the first 100 articles that appear from the search is the most relevant. Thus, the researcher used this approach for this review. Additionally, the researcher used a lateral searching technique to identify additional studies [40]. This included finding studies through relevant articles listed, checking reference lists and tracking citations. Electronic search results were downloaded into Covidence [38], a software to assist in screening and removing duplicate articles.

Table 1. Systematic Review Search Terms Used.

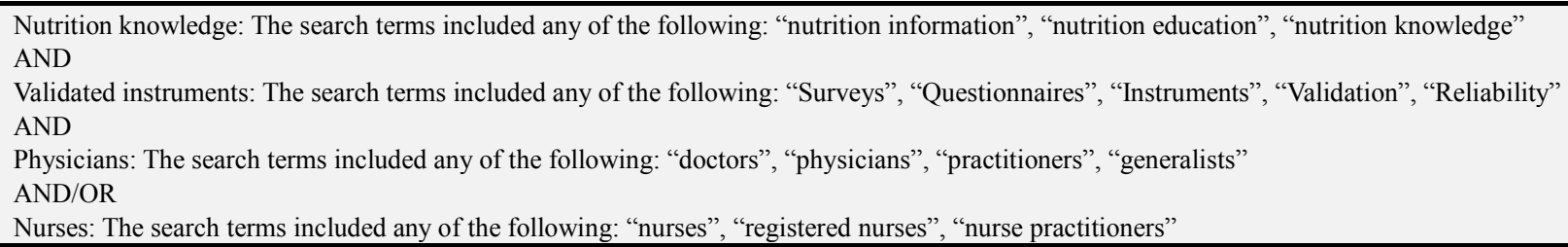

\subsection{Inclusion Criteria}

Studies were included if they were descriptive, in the English language, available in full-text and aimed at identifying nutrition knowledge of physicians and nurses. Participants had to be 18 years or older and physicians and/or nurses. Studies had to utilize validated instruments to identify nutrition knowledge. Primary outcomes had to include 
measurement of nutrition knowledge.

\subsection{Exclusion Criteria}

Conference abstracts, posters, books, studies that included an intervention, published protocols, non-validated instruments, health professionals/students other than physicians and nurses, outcomes not identifying nutrition knowledge and studies reported in languages other than English were excluded.

\subsection{Data Extraction}

All titles and abstracts were screened independently by two reviewers (J. Z. and J. G.) and full-text studies that were considered relevant were included for further review. Two reviewers (J. Z. and J. G. or J. M. A) independently reviewed all full-text articles and any discrepancies were resolved with consensus. The primary discussion surrounded the instruments and identification of nutrition knowledge. Data from the studies that satisfied the eligibility criterion were collected onto Microsoft Excel [39]: 1) first author's last name, 2) quality and risk of bias, 3) location, participants and size, 4) nutrition knowledge instrument, 5) validation/reliability of instrument, 6) nutrition knowledge outcomes, 7) areas of nutrition knowledge and 8) demographic factors and associations with nutrition knowledge.

\subsection{Quality and Risk of Bias Assessment}

Quality and risk of bias assessment of the studies used in this review followed the Academy of Nutrition and Dietetics (AND)'s Quality Criteria Checklist. This checklist consisted of two parts: relevance and validity. The first part, relevance, is defined by four parts and is used to determine a study's usefulness to the nutrition profession. If the responses to all four question were "yes" the researcher progressed to the validation questions. However, if the response to any of the four questions was "no", the article was removed from this review. The second part, validity, is defined by 10 domain questions and is used to determine the quality and risk of bias of each article. The 10 domain questions address the article's research question, subject selection, study population, withdrawals, blinding, intervention/exposure, outcomes, analysis, conclusion of support, and likelihood of bias [40, 41]. A complete description of each criterion is found in the AND's Evidence Analysis Manual [41].

For the validity part of the checklist, responses were either 0 (yes), 1 (no), 2 (unclear), or 3 (not applicable). If responses to at least five validity criteria were yes $(0)$, the article was deemed high quality $(+)$. If responses to at least six criteria were no (1), the article was deemed low quality (-). If determined low quality, the article was removed from further analysis. If responses to four validity criteria were no (1) or unclear (2), the article was determined neutral $(\Theta)$ [41].

Three researchers (J. Z, J. M. A and S. S.) independently reviewed the articles and critically evaluated them based on the Quality Criteria Checklist. Inter-rater reliability was then determined using a quadratic weighted Cohen's kappa to account for the degree of disagreement among raters [42]. Each reviewer's response to each question of the Quality Criteria Checklist was entered into SPSS v26 [43] to determine inter-rater reliability. Interpretation of the Cohen kappa results were as follows: values $\leq 0$ indicate no agreement; $0.01-0.20$ indicate none to slight agreement; 0.21-0.40 indicate fair agreement; $0.41-0.60$ indicate moderate agreement; $0.61-0.80$ indicate substantial agreement; and 0.81-1.00 indicate almost perfect agreement [42].

\subsection{Quality and Risk of Bias Assessment}

Considering the heterogeneity of the studies, a descriptive analytical approach was deemed acceptable for the quantitative data as opposed to a statistical or meta-analytical approach.

\section{Results}

\subsection{Search Results}

A total of 764 articles was identified initially after removal of duplicates. A total of 33 peer-reviewed articles was accepted as seen in Figure 1.

\subsection{Characteristics of Studies}

Studies were conducted in the United States $(n=7)[29,44-$ 49], Saudi Arabi ( $n=4)$ [50-53], Australia $(n=3)$ [54-56], Canada $(n=3)$ [57-59], Israel $(n=2)[60,61]$, Korea $(n=2)[62$, 63], Turkey $(\mathrm{n}=2)$ [64, 65], Austria $(\mathrm{n}=1)$ [66], Denmark, Sweden, and Norway $(n=1)$ [67] Ethiopia $(n=1)$ [68], Finland $(n=1)$ [31], Ghana $(n=1)$ [30], Greece $(n=1)$ [69], Qatar $(n=1)$ [70], South Africa $(n=1)$ [32], Sub-Saharan Africa $(n=1)$ [71], and Taiwan $(n=1)$ [72]. Participants in these studies were either physicians $(\mathrm{n}=12)[44,49-51,53,58,59,64,69-72]$ or nurses $(n=19)$ [29-32, 45-48, 52, 54-57, 60-63, 65, 66]. Two studies included both physicians and nurses [67, 68]. The smallest sample size was 59 [51] and the largest sample size was 4, 512 [67], the average sample size was 350 participants. Response rates for the number of instruments received varied greatly across all 33 studies (15\% to $100 \%)$ [47, 53, 54].

All studies sought to determine nutrition knowledge of physicians and/or nurses. Studies also associated the nutrition knowledge with demographic variables such as gender, years of practice, education level, and/or specialty field $(n=24)$ [29, $30,44-48,50,52-56,60-67,69,70,72]$.

\subsection{Nutrition Knowledge Instruments}

Nutrition knowledge instruments were either adapted and modified $(\mathrm{n}=22)$ [32, 45, 47-58, 60-64, 67, 69, 70] or developed for the study $(\mathrm{n}=11)$ [29-31, 44, 46, 59, 65, 66, 68, $71,72]$. The methods used to validate these instruments were face and/or content validity among content matter experts such as dietitians, physicians, and/or nurses. The nutrition knowledge portion of the instruments ranged from three 
items [57] to 50 items [29, 47, 48] and varied among multiple-choice $(\mathrm{n}=25)$ [30, 32, 44-54, 56, 59, 60, 63-66, 68-72], true or false $(\mathrm{n}=4)[30,49,54,62]$ and/or Likert scales $(n=7)[31,55,57,58,61,67,71]$. Four studies administered the instrument online $[49,53,55,71]$ while the other studies used paper-based instruments.

The instruments used focused on general nutrition knowledge such as food and nutrition principles, nutrient deficiencies, malnutrition, practices of nutrition assessment, nutrition-related resources, general knowledge about weight

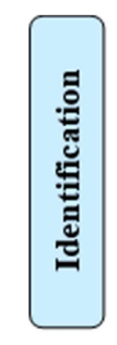

$$
\begin{aligned}
& \text { Records identified through database } \\
& \text { searching } \\
& (\mathrm{n}=1053)
\end{aligned}
$$
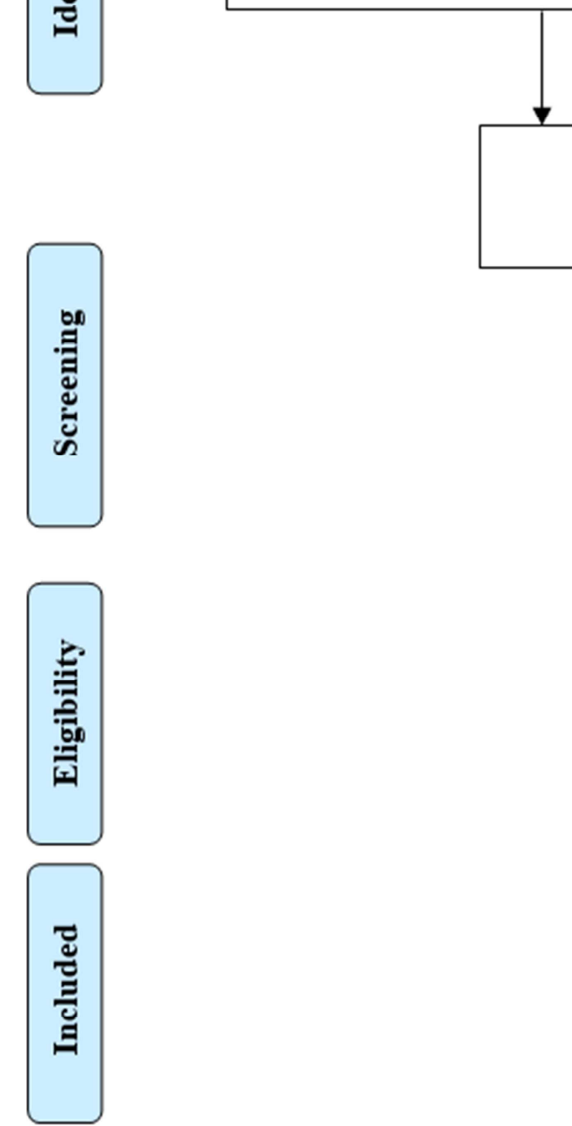

Records after duplicates removed $(\mathrm{n}=304)$

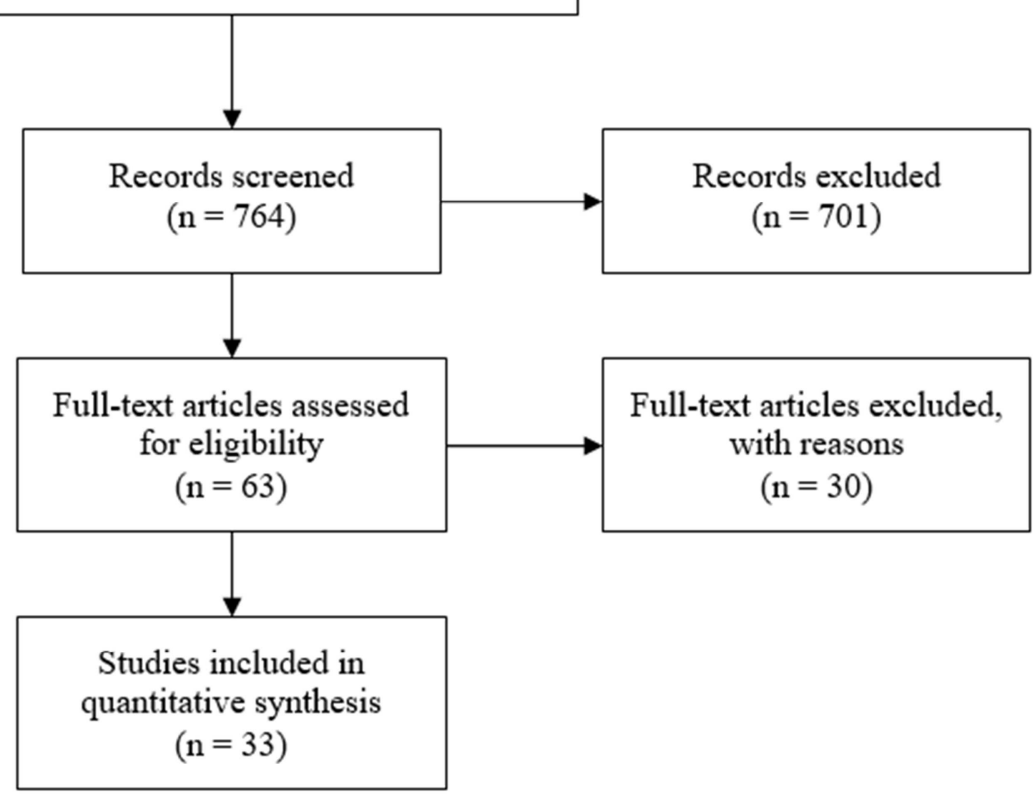

Figure 1. Article Extraction Process

\subsection{Nutrition Knowledge Outcomes}

Mean percentages of nutrition knowledge ranged from $32.5 \%$ correct [48] to $72 \%$ correct [31]. Across the studies, the following nutrition areas were the most known ( $>70 \%$ of respondents answered the questions correctly): nutrition for patients in critical care condition $(n=7)[45,52,55,57,58,63$, 65]; nutrition throughout the life span $(n=5)[29,31,60,61$, 66]; food sources and impact on health $(\mathrm{n}=8)[30,49-51,53$, $59,64,71]$; identification of fat sources $(n=3)[44,56,70]$; micronutrient deficiencies and impact on health $(n=7)[29,32$, management, nutrition in the life cycle, and the role of diet and disease. For studies that focused on nutrition knowledge, an overall nutrition knowledge score was calculated. Each correct answer was assigned one point, with the maximum score being the number of items in the questionnaire. Three studies grouped nutrition knowledge scores into categories: poor $(<10)$, moderate $(10-14)$, or very good (15-20) [70]; poor $(<8)$, mediocre $(9-12)$, good $(13-16)$, or very good $(17-20)[64]$ and inadequate $(\leq 12)$ or expert $(\geq 12)$ [69].

Additional records identified through other sources $(\mathrm{n}=15)$ 
Table 2. Results of Articles included in this Review $(n=33)$.

\begin{tabular}{|c|c|c|c|c|c|}
\hline Author (year) & $\begin{array}{l}\text { Location/ } \\
\text { Population }\end{array}$ & $\begin{array}{l}\text { Number of } \\
\text { knowledge } \\
\text { items }\end{array}$ & $\begin{array}{l}\text { Mean } \\
\text { Percentage } \\
\text { Correct }\end{array}$ & Areas of knowledge & $\begin{array}{l}\text { Demographics and Increased } \\
\text { nutrition knowledge }\end{array}$ \\
\hline Alkhaldy (2019) & $\begin{array}{l}\text { Saudi Arabia } \\
\text { Physicians }(\mathrm{N}=117)\end{array}$ & 18 & $50 \%$ & $\begin{array}{l}\text {-Most correct responses: Fat and salt } \\
\text { intake; risk factors } \\
\text {-Least correct responses: Smoking and } \\
\text { cardiac risk }\end{array}$ & $\begin{array}{l}\text { Significant associations among } \\
\text { older physicians }(p=0.01), 3 \text { or } \\
\text { more years of employment } \\
(p=0.04) \text {, higher education level } \\
(p<0.01)\end{array}$ \\
\hline Al-Numair (2004) & $\begin{array}{l}\text { Saudi Arabia } \\
\text { Physicians }(\mathrm{N}=59)\end{array}$ & 16 & $51.7 \%$ & $\begin{array}{l}\text {-Most correct responses: folate to } \\
\text { prevent neural tube defects; nutrients } \\
\text { to prevent thrombosis } \\
\text {-Least correct responses: Excess } \\
\text { protein and calcium; soluble fiber and } \\
\text { blood cholesterol }\end{array}$ & None reported \\
\hline $\begin{array}{l}\text { Al-Schwaiyat } \\
(2013)\end{array}$ & $\begin{array}{l}\text { Saudi Arabia } \\
\text { Nurses }(N=200)\end{array}$ & 31 & $58.8 \%$ & $\begin{array}{l}\text {-Most correct responses: nutrition and } \\
\text { diabetes; fiber diet and obesity risk } \\
\text {-Least correct responses: reducing fat } \\
\text { intake }\end{array}$ & $\begin{array}{l}\text { No significant association, but } \\
\text { female nurses had higher scores } \\
\text { compared to male nurses ( } 56 \% \text { vs } \\
51 \%)\end{array}$ \\
\hline Al-Zahrani (2009) & $\begin{array}{l}\text { Saudi Arabia } \\
\text { Physicians }(\mathrm{N}=125)\end{array}$ & 16 & $52.1 \%$ & $\begin{array}{l}\text { Most correct responses: Role of } \\
\text { vitamins and minerals; fruits, } \\
\text { vegetables and cancer risk }\end{array}$ & $\begin{array}{l}\text { Age and years in practice inversely } \\
\text { correlated }(\mathrm{p}<0.01)\end{array}$ \\
\hline Ameh (2019) & $\begin{array}{l}\text { Sub-Saharan Africa } \\
\text { Physicians }(\mathrm{N}=174)\end{array}$ & 16 & $63 \%$ & $\begin{array}{l}\text {-Most correct responses: cardiac } \\
\text { prevention for obesity; salt and fat } \\
\text { intake } \\
\text {-Least correct responses: smoking and } \\
\text { cardiac risk }\end{array}$ & None reported \\
\hline Bauer (2015) & $\begin{array}{l}\text { Austria } \\
\text { Nurses }(\mathrm{N}=458)\end{array}$ & 19 & $65.6 \%$ & $\begin{array}{l}\text {-Most correct responses: consequences } \\
\text { and signs of malnutrition } \\
\text {-Least correct responses: treating } \\
\text { malnutrition; body mass index }\end{array}$ & $\begin{array}{l}\text { No differences for gender }(p=0.80) \\
\text { or age }(p=0.25) \text {. } \\
\text { Significant associations for } \\
\text { practicing more than } 6 \text { years } \\
(p<0.001)\end{array}$ \\
\hline Boaz (2013) & $\begin{array}{l}\text { Israel } \\
\text { Nurses }(N=106)\end{array}$ & 18 & $51.9 \%$ & $\begin{array}{l}\text {-Most correct responses: hemoglobin } \\
\text { and elders; nutrition requirements and } \\
\text { trauma } \\
\text {-Least correct responses: feeding and } \\
\text { fistulas; body mass index }\end{array}$ & $\begin{array}{l}\text { No associations with age, years of } \\
\text { experience, or gender. } \\
\text { Positive statistical association in } \\
\text { length of time from migrating to } \\
\text { Israel }(r=0.21 ; \mathrm{p}=0.09)\end{array}$ \\
\hline Crogan (2000) & $\begin{array}{l}\text { United States } \\
\text { Nurses }(\mathrm{N}=105)\end{array}$ & 50 & $65 \%$ & $\begin{array}{l}\text { Most correct responses: nutritional } \\
\text { deficiencies; protein-calorie } \\
\text { malnutrition }\end{array}$ & $\begin{array}{l}\text { No associations of formal nutrition } \\
\text { education }\end{array}$ \\
\hline Daradkeh (2012) & $\begin{array}{l}\text { Qatar } \\
\text { Physicians }(\mathrm{N}=136)\end{array}$ & 20 & $63.9 \%$ & $\begin{array}{l}\text {-Most correct responses: folate to } \\
\text { prevent neural tube feeding; BMI } \\
\text { categories } \\
\text {-Least correct responses: Fats in eggs; } \\
\text { glycemic index of foods }\end{array}$ & $\begin{array}{l}\text { Significant associations of males, } \\
\text { specialized in the field or had }>10 \\
\text { years post university education } \\
(p<0.05)\end{array}$ \\
\hline Duerksen (2015) & $\begin{array}{l}\text { Canada } \\
\text { Physicians and } \\
\text { residents }(\mathrm{N}=428)\end{array}$ & 4 scenarios & $53 \%$ & $\begin{array}{l}>50 \% \text { of providers identified the } \\
\text { correct nutrition solution }\end{array}$ & None reported \\
\hline Duerksen (2016) & $\begin{array}{l}\text { Canada } \\
\text { Nurses }(\mathrm{N}=345)\end{array}$ & 3 scenarios & $64 \%$ & $\begin{array}{l}>60 \% \text { of nurses identified the correct } \\
\text { nutrition solution }\end{array}$ & None reported \\
\hline Endevelt (2009) & $\begin{array}{l}\text { Israel } \\
\text { Nurses }(\mathrm{N}=159)\end{array}$ & 8 & $69 \%$ & $\begin{array}{l}\text {-Most correct responses: calcium and } \\
\text { elders; importance of calories } \\
\text {-Least correct responses: Alzheimer's } \\
\text { disease and obesity and cholesterol }\end{array}$ & $\begin{array}{l}\text { Significant associations of nurses } \\
\text { younger than } 40 \text { years of age } \\
(p=0.04)\end{array}$ \\
\hline Flynn (2003) & $\begin{array}{l}\text { United States } \\
\text { Internists and } \\
\text { cardiologists } \\
(\mathrm{N}=639)\end{array}$ & 9 & $31 \%$ & $\begin{array}{l}\text { Least correct responses: fats in olive } \\
\text { and canola oils; diets impact on cardiac } \\
\text { disease }\end{array}$ & $\begin{array}{l}\text { Significant associations of } \\
\text { cardiologists compared to internal } \\
\text { medicine specialists }(\mathrm{p}<0.001)\end{array}$ \\
\hline $\begin{array}{l}\text { Grammatikopoulou } \\
\text { (2019) }\end{array}$ & $\begin{array}{l}\text { Greece } \\
\text { Physicians }(\mathrm{N}=115)\end{array}$ & 20 & $70 \%$ & $\begin{array}{l}\text { Least correct responses: macronutrient } \\
\text { calories; complications of refeeding } \\
\text { syndrome }\end{array}$ & $\begin{array}{l}\text { Significant associations of } \\
\text { attending continuing education } \\
\text { about nutrition }(\mathrm{p}=0.002)\end{array}$ \\
\hline Harkin (2019) & $\begin{array}{l}\text { United States } \\
\text { Physicians }(\mathrm{N}=236)\end{array}$ & 10 & $70 \%$ & $\begin{array}{l}\text {-Most correct responses: foods impact } \\
\text { on chronic disease } \\
\text {-Least correct responses: foods high in } \\
\text { soluble fiber and omega } 3\end{array}$ & No associations \\
\hline Hu (1997) & $\begin{array}{l}\text { Taiwan } \\
\text { Physicians }(\mathrm{N}=326)\end{array}$ & 26 & $58.7 \%$ & $\begin{array}{l}\text {-Most correct responses: nutrient } \\
\text { functions; pregnancy nutrition } \\
\text {-Least correct responses: nutrition }\end{array}$ & $\begin{array}{l}\text { Significant associations of female } \\
\text { physicians, } 35 \text { years and younger, } \\
\text { non-smokers }(\mathrm{p}<0.05)\end{array}$ \\
\hline
\end{tabular}




\begin{tabular}{|c|c|c|c|c|c|}
\hline Author (year) & $\begin{array}{l}\text { Location/ } \\
\text { Population }\end{array}$ & $\begin{array}{l}\text { Number of } \\
\text { knowledge } \\
\text { items }\end{array}$ & $\begin{array}{l}\text { Mean } \\
\text { Percentage } \\
\text { Correct }\end{array}$ & Areas of knowledge & $\begin{array}{l}\text { Demographics and Increased } \\
\text { nutrition knowledge }\end{array}$ \\
\hline Ilmonen (2012) & $\begin{array}{l}\text { Finland } \\
\text { Nurses }(\mathrm{N}=650)\end{array}$ & 33 & $72 \%$ & $\begin{array}{l}\text { assessment } \\
\text { Most correct responses: food allergies; } \\
\text { vitamin D supplementation }\end{array}$ & None reported \\
\hline Kable (2015) & $\begin{array}{l}\text { Australia } \\
\text { Nurses }(\mathrm{N}=79)\end{array}$ & 10 & $68 \%$ & $\begin{array}{l}\text { Least correct responses: weight } \\
\text { management and physical activity }\end{array}$ & $\begin{array}{l}\text { No associations of where nurses } \\
\text { worked (rural vs urban) }\end{array}$ \\
\hline Kgaphola (1997) & $\begin{array}{l}\text { South Africa Nurses } \\
(\mathrm{N}=99)\end{array}$ & 40 & $35 \%$ & $\begin{array}{l}\text {-Most correct responses: functions and } \\
\text { sources of nutrients; meat substitutions } \\
\text {-Least correct responses: causes of } \\
\text { weight gain; reading nutrition labels }\end{array}$ & None reported \\
\hline Kim (2009) & $\begin{array}{l}\text { Korea } \\
\text { Nurses }(\mathrm{N}=221)\end{array}$ & 4 & $48.4 \%$ & $\begin{array}{l}\text {-Most correct responses: appropriate } \\
\text { nutrition support; energy requirements } \\
\text {-Least correct responses: interpreting } \\
\text { blood laboratory results; body mass } \\
\text { index }\end{array}$ & $\begin{array}{l}\text { Significant associations of those } \\
\text { nurses who wanted nutrition } \\
\text { education compared to those who } \\
\text { do not want it }(\mathrm{p}<0.05)\end{array}$ \\
\hline Lindseth (1990) & $\begin{array}{l}\text { United States } \\
\text { Nurses }(\mathrm{N}=176)\end{array}$ & 50 & $32.5 \%$ & Not stated & $\begin{array}{l}\text { Significant associations between } \\
\text { years since graduating }(>6 \text { years }) \\
(\mathrm{p}<0.005) \text { and practice area } \\
(\mathrm{p}<0.001)\end{array}$ \\
\hline Lindseth (1994) & $\begin{array}{l}\text { United States } \\
\text { Nurses }(\mathrm{N}=71)\end{array}$ & 50 & $64 \%$ & $\begin{array}{l}\text {-Most correct responses: cultural } \\
\text { nutrition; regulatory considerations } \\
\text {-Least correct responses: digestion, } \\
\text { absorption, and metabolism of } \\
\text { nutrients }\end{array}$ & $\begin{array}{l}\text { No associations of employment or } \\
\text { education levels }(\mathrm{p}>0.05) \text {. } \\
\text { Significant associations of } \\
\text { cultural/regulator agency }(\mathrm{p}=0.03)\end{array}$ \\
\hline Martin (2014) & $\begin{array}{l}\text { Australia } \\
\text { Nurses }(\mathrm{N}=181)\end{array}$ & 18 & $56.4 \%$ & $\begin{array}{l}\text { Respondents differed in how to care } \\
\text { for chronically ill patients }\end{array}$ & $\begin{array}{l}\text { Significant associations of } \\
\text { nurses }>50 \text { years of age }(\mathrm{p}=0.016) \text {; } \\
\text { nurses with }>7 \text { years of experience } \\
(\mathrm{p}=0.001) ; \text { nurses of normal or } \\
\text { underweight }(\mathrm{p}=0.036))\end{array}$ \\
\hline Mogre (2017) & $\begin{array}{l}\text { Ghana } \\
\text { Nurses }(N=104)\end{array}$ & 26 & $54 \%$ & $\begin{array}{l}\text {-Most correct responses: preventing } \\
\text { malnutrition } \\
\text {-Least correct responses: signs and } \\
\text { symptoms of malnutrition }\end{array}$ & $\begin{array}{l}\text { Significant associations among } \\
\text { years as a nurse }(\mathrm{p}<0.001) \text { and } \\
\text { trained in nutrition guidelines } \\
(\mathrm{p}=0.004)\end{array}$ \\
\hline Mowe (2008) & $\begin{array}{l}\text { Denmark, Sweden, } \\
\text { Norway } \\
\text { Physicians and } \\
\text { nurses }(\mathrm{N}=4512)\end{array}$ & 4 & $58 \%$ & $\begin{array}{l}\text { Respondents who had more positive } \\
\text { attitudes about their nutrition } \\
\text { knowledge provided correct answers }\end{array}$ & $\begin{array}{l}\text { Significant associations of } \\
\text { Denmark respondents compared to } \\
\text { other countries }(\mathrm{p}<0.001)\end{array}$ \\
\hline Ozcelik (2007) & $\begin{array}{l}\text { Turkey } \\
\text { Physicians }(\mathrm{N}=210)\end{array}$ & 20 & $48.1 \%$ & $\begin{array}{l}\text {-Most correct responses: importance } \\
\text { of fruits and vegetables; energy value } \\
\text { of fat } \\
\text {-Least correct responses: alcohol and } \\
\text { soluble fiber's impact on cholesterol }\end{array}$ & $\begin{array}{l}\text { Significant associations of older } \\
(\mathrm{p}<001) \text {; who specialized } \\
(\mathrm{p}<0.05) \text {; practiced in the field for } \\
\text { a length of time }(\mathrm{p}<0.001)\end{array}$ \\
\hline Park (2011) & $\begin{array}{l}\text { Korea } \\
\text { Nurses }(\mathrm{N}=506)\end{array}$ & 42 & $58.4 \%$ & $\begin{array}{l}\text { Least correct responses: dietary } \\
\text { approaches to reducing/preventing } \\
\text { chronic diseases }\end{array}$ & $\begin{array}{l}\text { Significant associations with }>11 \\
\text { years of experience }(p<0.05) \\
\text { master's degree }(\mathrm{p}<0.05)\end{array}$ \\
\hline Perry (1997) & $\begin{array}{l}\text { United States } \\
\text { Nurses }(\mathrm{N}=97)\end{array}$ & 14 & $40-50 \%$ & $\begin{array}{l}\text {-Most correct responses: number of } \\
\text { days on clear liquids } \\
\text {-Least correct responses: calorie needs } \\
\text { for enteral nutrition }\end{array}$ & $\begin{array}{l}\text { Significant associations who were } \\
\text { at higher grades }(\mathrm{p}<0.05)\end{array}$ \\
\hline Schaller (2005) & $\begin{array}{l}\text { Australia } \\
\text { Nurses }(\mathrm{N}=103)\end{array}$ & 48 & $60.2 \%$ & $\begin{array}{l}\text {-Most correct responses: } \\
\text { vitamins/minerals role; fats in food } \\
\text {-Least correct responses: non-essential } \\
\text { amino acids; lactating nutrient needs }\end{array}$ & $\begin{array}{l}\text { Significant associations of older } \\
\text { nurses }(>36 \text { years of age }) \\
((p=0.004) ; \text { more experience }(>10 \\
\text { years })(p=0.024) ; \text { held } \\
\text { diploma/general nurse training } \\
(p=0.029)\end{array}$ \\
\hline Stanek (1991) & $\begin{array}{l}\text { United States } \\
\text { Nurses }(\mathrm{N}=95)\end{array}$ & 15 & $60 \%$ & $\begin{array}{l}\text { Respondents had difficulty with all } \\
\text { questions }\end{array}$ & $\begin{array}{l}\text { No associations between age, } \\
\text { years of experience, level of } \\
\text { nutrition education }\end{array}$ \\
\hline Tafese (2015) & $\begin{array}{l}\text { Ethiopia } \\
\text { Nurses, trained } \\
\text { health care workers, } \\
\text { physicians }(\mathrm{N}=355)\end{array}$ & 13 & $55.5 \%$ & $\begin{array}{l}\text {-Most correct responses: using } \\
\text { malnutrition chart correctly } \\
\text {-Least correct responses: detecting } \\
\text { and treating malnutrition }\end{array}$ & None reported \\
\hline Temple (1999) & $\begin{array}{l}\text { Canada } \\
\text { Physicians (N=84) }\end{array}$ & 16 & $63.1 \%$ & $\begin{array}{l}\text {-Most correct responses: folate to } \\
\text { prevent neural tube defects; thiamin } \\
\text { deficiency in alcoholics } \\
\text {-Least correct responses: excess }\end{array}$ & None reported \\
\hline
\end{tabular}




\begin{tabular}{llllll}
\hline Author (year) & $\begin{array}{l}\text { Location/ } \\
\text { Population }\end{array}$ & $\begin{array}{l}\text { Number of } \\
\text { knowledge } \\
\text { items }\end{array}$ & $\begin{array}{l}\text { Mean } \\
\text { Percentage } \\
\text { Correct }\end{array}$ & Areas of knowledge & $\begin{array}{l}\text { Demographics and Increased } \\
\text { nutrition knowledge }\end{array}$ \\
\hline & & & $\begin{array}{l}\text { protein and calcium loss; soluble fiber } \\
\text { and impact on cholesterol } \\
\text {-Most correct responses: symptoms of }\end{array}$ & $\begin{array}{l}\text { No associations of nutrition } \\
\text { education ( }=0.289) . \text { Significant } \\
\text { catheter infection; enteral access types } \\
\text { associations of those who provide } \\
\text { nutritional assessment (p=0.012); } \\
\text { had a graduate degree (p=0.000). }\end{array}$ \\
\hline
\end{tabular}

\subsection{Associations Between Nutrition Knowledge and Demographics}

Twenty-five studies determined associations between nutrition knowledge and demographic variables such as age, gender and educational level. Higher nutrition knowledge was associated with age $(n=7)[50,53,55,56,61,64,72]$; gender $(n=2)[70,72]$; immigrating to the country $(n=1)[60]$; years in practice $(\mathrm{n}=10)[30,48,50,53,55,56,62,64,66$, 70]; held an advanced degree $(n=4)[53,56,62,65]$; pursued nutrition education and/or training $(\mathrm{n}=4)[30,63,65,69]$; and specialized in an area $(n=5)[44,45,48,64,70]$. However, discrepancies existed among age as two studies found associations between nurses who were $<40$ years of age [61, 72] and three studies showed associations between nurses who were $>50$ years of age and had higher nutrition knowledge [55, 56, 64]. One study did not specify the age of the nurses and the association [50]. The same discrepancy existed between gender and higher nutrition knowledge in which one study identified an association between females having higher nutrition knowledge [72] and

one study identifying that males had higher nutrition knowledge [70]. Ten studies found no associations between demographics and nutrition knowledge [29, 46, 47, 49, 52, 54, $60,65,66,69]$ and two studies identified associations between providing nutrition in a clinical setting to higher nutrition knowledge $[60,67]$ as seen in table 2 .

\subsection{Quality and Risk of Bias}

Thirty-two studies were considered high-quality as more than 5 out of the 10 validation questions had a response of yes. One study was considered neutral as 4 out of the 10 validation questions had a response of no or unclear. The most common factors that influenced the validity of these studies were the lack of different study groups and lack of studies reporting statistics with levels of significance. The overall Cohen kappa scores was 0.76 , which demonstrates substantial agreement among the researchers [42].

\section{Discussion}

This systematic narrative review sought to identify the nutrition knowledge within specific areas through validated instruments. Studies used validated surveys that were either adopted and modified from prior surveys or else initially developed. Instruments, though, varied between studies as well as the type of nutrition knowledge that was to be assessed.
Mean nutrition knowledge percentages ranged from a low of $32 \%$ correct responses to a high of $72 \%$ correct responses.

All studies utilized validated instruments that were either in its original form or adapted and modified from previous studies to assess nutrition knowledge [74]. For those studies that designed their own instrument, previous nutrition knowledge instruments were not appropriate based on their research objectives. Although there are advantages to composing study-specific instruments, such as including only questions that are relevant to the purpose of the study, utilizing a non-validated questionnaire may lead to results that are difficult to compare with those obtained from previous studies [21]. It is also important to recognize that although an instrument may have been validated in its original state, a further validation study is necessary upon altering the original form [21]. For nutrition knowledge instruments specifically, it is likely that constructs will need to be modified due to new dietary recommendations being published [21]. Additionally, because studies were conducted across different continents and countries, instruments needed to be modified to reflect that specific population. Based on the studies within this systematic narrative review, if studies were utilizing modified and adapted instruments, a form of face, content, or construct validation took place among content matter experts. Once those instruments were further modified, they had undergone pilot studies using a sample of the population to ensure the instrument was not only valid, but also reliable. However, few studies illustrated the mechanisms to validate and ensure reliability of these modified instruments, thus it is not known if these instruments were valid and reliable among the population. The same strategies (e.g. content matter experts and pilot study among sample population) were used for newly developed instruments. Although, these studies also lacked in providing the validity and reliability results. Because most of the instruments were adapted, some validity may have been lost upon modification if the adapted instrument did not undergo an additional validation study. Nevertheless, although the instruments may have been adapted to accurately reflect the chosen population of physicians or nurses, the content of the instruments seemed to provide insight of the health professionals' nutrition knowledge.

With regards to the instruments in general, it is important to recognize the importance of representativeness and validity in relation to response rate. Representativeness refers to how well the sample population compares with the actual population of interest [75]. In the case of this systematic narrative review, it is important to inquire whether the sample 
size of the physicians and nurses utilized is adequate to represent the actual population of physicians and nurses in each respective geographical location. Sample sizes and response rates varied greatly across the studies, which may be in part due to different recruitment methods utilized. For the studies that mentioned a response rate, the average response rate was $58 \%$ and ranged from $15 \%$ [54] to $100 \%$, leaving a nonresponse rate of $42 \%$ [47]. A response rate of approximately $60 \%$ or more is indicated to be the goal of researchers [75], suggesting that the studies utilized in this review, on average, had appropriate response rates. Nonetheless, research has shown that studies with lower response rates are sometimes capable of yielding more accurate results than studies with high response rates [76]. Therefore, there is not a direct correlation between response rate and validity, indicating that low response rates in some of the studies do not automatically mean the study results have lower validity [76].

Physicians and nurses had higher knowledge regarding the topics of nutrition for critical care patients, nutrition during the life span, the role of vitamins and minerals, and/or food sources/macronutrients and the impact on health. On the other hand, nutrition knowledge among physicians and nurses was lower in the topics of nutrition management for chronic diseases/conditions, digestion and absorption and metabolism of foods, and/or presence of macronutrients in foods. Furthermore, physicians and nurses who were specialists (e.g. geriatric or cardiologists), had more years of practice experience, had higher nutrition knowledge compared to physicians and nurses who were not specialists and had less years of practice experience.

Mean scores on the nutrition knowledge instruments ranged from poor, $32.5 \%$ correct [48], to fair, $72 \%$ correct [31], indicating that there is a gap in nutrition knowledge among physicians and nurses. Further analysis from these studies illustrated that certain demographic differences such as specializing in an area, interest in nutrition education, years in practice or positive attitudes towards nutrition or providing nutrition information to patients resulted in higher nutrition knowledge scores overall. Some factors such as age and gender resulted in different nutrition knowledge scores, in which studies showed that male [70] or female [72] physicians and nurses or those younger than 40 years of age $[61,72]$ or those older than 50 years of age had higher nutrition knowledge scores $[55,56,64]$. Even though there may have been a discrepancy between age and gender from the studies, commonalities were that those physicians and nurses held positive attitudes towards nutrition, provided nutrition information to their patients, specialized in an area, took more nutrition classes in their professional studies and/or pursued continuing education specifically in nutrition $[61,70,72]$. Thus, it is difficult to conclude age or gender is directly associated with knowledge as other mediators may play a role in one's knowledge.

Although physicians and nurses had high nutrition knowledge scores from certain topics such as importance of nutrition during critical care and role of vitamins and minerals, they had low nutrition knowledge scores related to digestion, absorption and metabolism of nutrients and proper nutrition for chronic diseases/conditions. Nutrition is vital to the maintenance of health and the prevention of many diseases [3-5]. Nutrition interventions have been shown to decrease morbidity, mortality, human suffering, and medical costs [5, 77]. Adequate nutrition knowledge is necessary for patients to adopt appropriate dietary habits and is predictive of dietary intake change [78]. If health professionals lack appropriate nutrition knowledge themselves, they subsequently lack the expertise to provide high quality nutrition care and education to patients.

Lack of nutrition knowledge among physicians and nurses may in part be related to the extent of nutrition training in professional school, as well as in continuing education of health professionals. There is evidence that medical graduates do not receive sufficient training in nutrition throughout the world [5, 18, 25-27]. In 1985, the US National Academy of Sciences recommended a minimum of 25 hours of nutrition education in medical schools [79]. Three decades later, in 2015, only one-quarter of US professional schools meet those recommendations [26]. Similar scenarios have been observed in other countries, as well. For instance, two of the five medical schools in Greece do not possess a nutrition course [80]. Outside of the financial burden involved in implementing nutrition training in professional schools, a major obstacle is that there is a lack of specialized physicians and nurses who are trained in nutrition [80].

It is important to note that this systematic narrative review focused on physicians and nurses only. Therefore, it is not appropriate to suggest that there is a gap of nutrition knowledge among all health professionals conclusively. An additional limitation is that although all instruments were validated and created to assess nutrition knowledge, they did vary from study to study. Many of the studies used instruments that were adapted and modified, which, as mentioned previously, may have decreased the validity of the instrument if no further validation study was conducted. Nonetheless, the use of different instruments is also a strength of this review. Different instruments assessing the same concept provided diverse responses, yet still proved to show that nutrition knowledge was subpar across all the studies. An additional strength is that this systematic narrative review included no restriction on geographical location. This is important in that all studies on the topic of interest were considered, and lack of nutrition knowledge among health professionals is not only a problem nationwide but is also a problem globally.

\section{Conclusions}

This review provided insights to the areas of nutrition knowledge among physicians and nurses. Instruments used to assess nutrition knowledge varied but were shown to be validated and reliable. Further studies need to be conducted to assess nutrition knowledge of other health professionals outside of physicians and nurses, but it is evident that 
advanced nutrition education needs to be better integrated into professional schools and graduate programs. Additionally, continuing nutrition education should be required for all health professionals to ensure the most current nutrition recommendations are being implemented.

\section{Acknowledgements}

Thank you to Jessica Goldberg for assisting with the screening and full-text review of these articles. Somir Shreim who assisted with the quality and risk of bias analysis of the articles.

\section{References}

[1] World Health Organization. NCD mortality and morbidity. Global Health Observatory Data. http://apps.who.int/gho/data/node.main.A860. Published 2018. Retrieved December 152019.

[2] Global Action Plan: For the Prevention and Control of Noncommunicable Diseases 2013-2020. Geneva, Switzerland; 2020 .

[3] Khandelwal S, Kurpad A, Narayan KMV. Global non-communicable diseases - The nutrition conundrum. Front Public Heal. 2018; 6 (9): 1-3.

[4] Branca F, Lartey A, Oenema S, et al. Transforming the food system to fight non-communicable diseases. BMJ. 2019; 364. doi: 10.1136/bmj.1296.

[5] Nowson CA, O'Connell SL. Nutrition knowledge, attitudes, and confidence of Australian general practice registrars. J Biomed Educ. 2015; 2015: 1-6. doi: 10.1155/2015/219198.

[6] Wang X, Ouyang Y, Liu J, et al. Fruit and vegetable consumption and mortality from all causes, cardiovascular disease, and cancer: Systematic review and dose-response meta-analysis of prospective cohort studies. BMJ. 2014; 349 (July): 1-14. doi: 10.1136/bmj.g4490.

[7] Bélanger M, Poirier M, Jbilou J, Scarborough P. Modelling the impact of compliance with dietary recommendations on cancer and cardiovascular disease mortality in Canada. Public Health. 2014; 128 (3): 222-230. doi: 10.1016/j.puhe.2013.11.003.

[8] Adams KM, Lindell KC, Kohlmeier M, Zeisel SH. Status of nutrition education in medical schools. Am J Clin Nutr. 2006; 83 (4).

[9] Kardakis T, Jerdén L, Nyström ME, Weinehall L, Johansson H Implementation of clinical practice guidelines on lifestyle interventions in Swedish primary healthcare - a two-year follow up. BMC Health Serv Res. 2018; 18 (1): 1-13. doi: 10.1186/s12913-018-3023-z.

[10] Ball L, Desbrow B, Leveritt M. An exploration of individuals' preferences for nutrition care from Australi. Aust J Prim Health. 2014; 20: 113-120.

[11] Potter MB, Vu JD, Croughan-Minihane M. Weight management: What patients want from their primary care physicians. J Fam Pract. 2001; 50 (6): 513-518. http://www.ncbi.nlm.nih.gov/pubmed/11401737.

[12] Mowe M, Bosaeus I, Rasmussen H, Kondrup J, Unosson M.
Nutritional routines and attitudes among doctors and nurses in Scandinavia: A questionnaire based survey. J Clin Nutr. 2006; 25: 524-532.

[13] Wechsler H, Levine S, Idelson RK, Schor EL, Coakley E. The physician's role in health promotion revisited - A survey of primary care practitioners. N Engl J Med. 1996; 334 (15): 996-998.

[14] Smith S, Seeholzer EL, Gullett H, et al. Primary care residents' knowledge, attitudes, self-efficacy, and perceived professional norms regarding obesity, nutrition, and physical activity counseling. J Grad Med Educ. 2015; 7 (3): 388-394. doi: 10.4300/JGME-D-14-00710.1.

[15] Mogre V, Stevens FCJ, Aryee PA, Matorwmasen-Akkermans FL, Abubakari B, Scherpbier AJJA. Nutrition care practices, barriers, competencies and education in nutrition: A survey among Ghanaian medical doctors. Med Sci Educ. 2018; 28 (4): 815-824. doi: 10.1007/s40670-018-0591-9.

[16] Kris-etherton PM, Akabas SR, Bales CW, et al. The need to advance nutrition education in the training of health care professionals and recommended research to evaluate implementation. Am J Clin Nutr. 2018; 99 (August). doi: 10.3945/ajcn.113.073502.

[17] Association of American Medical Colleges. Medical School Graduation Questionnaire: 2018 All Schools Summary Report. 2018; $2019 \quad$ (March): 47. https://www.aamc.org/download/490454/data/2018gqallschool ssummaryreport.pdf.

[18] Abdollahi M, Houshiarrad A, Abtahi M, et al. The nutrition knowledge level of physicians, nurses and nutritionists in some educational hospitals. J Paramed Sci. 2013; 4 (Supplement): 106-114. doi: 10.22037/jps.v4i0.4151.

[19] Stotts NA, Englert DA, Crocker KS. Nutrient education in schools of nursing in the United States. Part 2: The status of nutrition education in schools of nursing. J Parenter Enter Nutr. 1987; 11 (4): 406-411. doi: 10.1177/0148607187011004406.

[20] Dumic A, Miskulin M, Pavlovic N, Orkic Z, Bilic-Kirin V, Miskulin I. The nutrition knowledge of Croatian general practitioners. J Clin Med. 2018; 7 (7): 178. doi: 10.3390/jcm7070178.

[21] Parmenter K, Wardle J. Evaluation and design of nutrition knowledge measures. J Nutr Educ. 2000; 32: 269-277.

[22] Axelson ML, Bringburn D. The measurement and conceptualization of nutrition knowledge. J Nutr Educ. 1992; 24 (5): 239-246.

[23] Sapp SG, Jensen HH. Reliability and validity of nutrition knowledge and diet-health awareness tests developed from the 1989-1991 Diet and health knowledge surveys. J Nutr Educ. 1997; 29: 63-72.

[24] Feren A, Torheim LE, Lillegaard ITL. Development of a nutrition knowledge questionnaire for obese adults. Food Nutr Res. 2011; 55.

[25] Devries S, Willett WC, Bonow RO. Nutrition education in medical school, residency training, and practice. J Am Med Assoc. 2019; 321 (14): 1351-1352. doi: 10.1155/2015/357627.

[26] Adams KM, Butsch WS, Kohlmeier M. The state of nutrition education at US medical schools. J Biomed Educ. 2015; 1-7. doi: $10.1155 / 2015 / 357627$. 
[27] DiMaria-Ghalili RA, Mirtallo JM, Tobin BW, Hark L, Van Horn L, Palmer CA. Challenges and opportunities for nutrition education and training in the health care professions: Intraprofessional and interprofessional call to action. Am J Clin Nutr. 2014; 99 (5): 1184-1193. doi: 10.3945/ajcn.113.073536.

[28] Aspry KE, Van Horn L, Carson JAS, et al. Medical nutrition education, training, and competencies to advance guideline-based diet counseling by physicians: A science advisory from the American Heart Association. Circulation. 2018; 137 (23): 821-841. doi: 10.1161/cir.0000000000000563.

[29] Crogan NL, Shultz JA. Comparing nutrition knowledge exam scores with reported nutrition topics of. J Nurses Staff Dev JNSD. 2000; 16 (6): 277-281.

[30] Mogre V, Yakubu A, Fuseini M, Amalba A, Aguree S. Nurses' knowledge and attitudes regarding malnutrition in children and its management in Ghana. Curationis. 2017; 40 (1): 1-8. doi: 10.4102/curationis.v40i1.1618.

[31] Ilmonen J, Isolauri E, Laitinen K. Nutrition education and counselling practices in mother and child health clinics: Study amongst nurses. J Clin Nurs. 2012; 21 (19-20): 2985-2994. doi: 10.1111/j.1365-2702.2012.04232.x.

[32] Kgaphola MS, Wodarski LA, Garrison MEB. Nutrition knowledge of clinic nurses in Lebowa, South Africa: Implications for nutrition services delivery. J Hum Nutr Diet. 1997; $10 \quad$ (5): $\quad 295-303 . \quad$ doi: 10.1046/j.1365-277X.1997.00063.x.

[33] Kazi AM, Khalid W. Questionnaire designing and validation. J Pak Med Assoc. 2012; 62 (5): 514-516.

[34] Lai PSM. Validating instruments of measure: Is it really necessary? Malaysian Fam Physician. 2013; 8 (1): 2-4.

[35] Garcia de Yebenes Prous M, Rodriguez SF, Carmona OL. Validation of questionnaires. Reumatol Clin. 2009; 5: 171-177.

[36] Moher D, Liberati A, Tetzlaff J, Altman D. Preferred Reporting Items for Systematic Reviews and Meta-Analyses: The PRISMA Statement. PLoS Med. 2009; 6 (7). doi: 10.1371/journal.pmed1000097.

[37] Higgins JPT, Thomas J, Chandler J, Cumpston M, Li T, Page MJ WV, ed. Cochrane Handbook for Systematic Reviews of Interventions. 6th ed. Cochrane; 2019. www.training.cochrane.org/handbook.

[38] Covidence Systematic Review Software. Melbourne, Australia: Veritas Health Health Innovation; 2018.

[39] Microsoft Excel. Version 1902. Redmond, WA: Microsoft Corp; 2018.

[40] Academy of Nutrition and Dietetics. Evidence Analysis Manual: Steps in the Academy Evidence Analysis Process. 2016.

[41] Mumby S, Leineweber M, Andrade J. The impact the smarter lunchroom movement strategies have on school children's healthy food selection and consumption: A systematic review. J Child Nutr Manag. 2018; 42 (2).

[42] Landis JR, Koch GG. The measurement of observer agreement for categorical data. Biometrics. 1977; 33 (1): 159-174. doi: $10.2307 / 2529310$.

[43] IBM SPSS Statistics for Windows, version 25 (IBM Corp., Armonk, N. Y., USA).
[44] Flynn M, Sciamanna C, Vigilante K. Inadequate physician knowledge of the effects of diet on blood lipids and lipoproteins. Nutr J. 2003; 2 (April 1998): 1-4. doi: 10.1186/1475-2891-2-19.

[45] Perry L. Fishing for understanding: Nurses knowledge and attitudes in relation to nutritional care. Int J Nurs Stud. 1997; 34 (6): 395-404.

[46] Stanek KL, Powell M, Betts N. Nutritional knowledge of nurses in long-term health care facilities. J Nutr Elder. 1991; 10 (3): 35-48. doi: 10.1300/J052v10n03.

[47] Lindseth G. Nutriton preparation and the geriatric nurse. West J Nurs Res. 1994; 16 (6): 692-703.

[48] Lindseth G. Evaluating rural nurses for preparation in implementing nutrition interventions. J Rural Heal. 1990; 6 (3): 231-245. doi: 10.1111/j.1748-0361.1990.tb00664.x.

[49] Harkin N, Johnston E, Mathews T, et al. Physicians' dietary knowledge, attitudes, and counseling practices: The experience of a single health care center at changing the landscape for dietary education. Am J Lifestyle Med. 2019; 13 (3): 292-300. doi: $10.1177 / 1559827618809934$

[50] Al-Zahrani AM, Al-Raddadi RM. Nutritional knowledge of primary health care physicians in Jeddah, Saudi Arabia. Saudi Med J. 2009; 30 (2): 284-287.

[51] Al-Numair KS. Nutrition knowledge of primary care physicians in Saudi Arabia. Pakistan J Nutr. 2004; 3 (6): 344-347. doi: 10.3923/pjn.2004.344.347.

[52] Al-Shwaiyat NM. Assessment of therapeutic nutritional knowledge of Jordanian nurses. Int J Nutr Food Sci. 2013; 2 (3) 142. doi: $10.11648 /$ j.ijnfs.20130203.18.

[53] Alkhaldy AA. Nutritional knowledge and self-reported nutritional practice against malnutrition among physicians in Jeddah, Saudi Arabia. Healthcare. 2019; 7 (4): 149. doi: 10.3390/healthcare 7040149 .

[54] Kable A, James C, Snodgrass S, et al. Nurse provision of healthy lifestyle advice to people who are overweight or obese. Nurs Heal Sci. 2015; 17 (4): 451-459. doi: 10.1111/nhs. 12214.

[55] Martin L, Leveritt MD, Desbrow B, Ball LE. The self-perceived knowledge, skills and attitudes of australian practice nurses in providing nutrition care to patients with chronic disease. Fam Pract. 2014; 31 (2): 201-208. doi: 10.1093/fampra/cmt070.

[56] Schaller C, James EL. The nutritional knowledge of Australian nurses. Nurse Educ Today. 2005; 25 (5): 405-412. doi: 10.1016/j.nedt.2005.04.002.

[57] Duerksen DR, Keller HH, Vesnaver E, et al. Nurses' perceptions regarding the prevalence, detection, and causes of malnutrition in Canadian hospitals: Results of a Canadian malnutrition task force survey. J Parenter Enter Nutr. 2016; 40 (1): 100-106. doi: 10.1177/0148607114548227.

[58] Duerksen DR, Keller HH, Vesnaver E, et al. Physicians' perceptions regarding the detection and management of malnutrition in Canadian hospitals: Results of a Canadian malnutrition task force survey. J Parenter Enter Nutr. 2015; 39 (4): 410-417. doi: 10.1177/0148607114534731.

[59] Temple NJ. Survey of nutrition knowledge of Canadian physicians. J Am Coll Nutr. 1999; 18 (1): 26-29. doi: 10.1080/07315724.1999.10718823. 
[60] Boaz M, Rychani L, Barami K, et al. Nurses and nutrition: A survey of knowledge and attitudes regarding nutrition assessment and care of hospitalized elderly patients. J Contin Educ Nurs. 2013; 44 (8): 357-364. doi: 10.3928/00220124-20130603-89.

[61] Endevelt R, Werner P, Goldman D, Karpati T. Nurses' knowledge and attitudes regarding nutrition in the elderly. $\mathrm{J}$ Nutr Heal Aging. 2009; 13 (6): 485-489.

[62] Park KA, Cho WI, Song KJ, Lee YS, Sung IS, Choi-Kwon SM. Assessment of nurses' nutritional knowledge regarding therapeutic diet regimens. Nurse Educ Today. 2011; 31 (2): 192-197. doi: 10.1016/j.nedt.2010.05.017.

[63] Kim H, Choue R. Nurses' positive attitudes to nutritional management but limited knowledge of nutritional assessment in Korea. Int Nurs Rev. 2009; 56 (3): 333-339. doi: 10.1111/j.1466-7657.2009.00717.x.

[64] Ozcelik AO, Surucuoglu MS, Akan LS. Survey on the nutrition knowledge level of turkish physicians: Ankara as a sample. Pakistan J Nutr. 2007; 6 (6): 538-542.

[65] Yalcin N, Cihan A, Gundogdu H, Ocakci A. Nutrition knowledge of nurses. Heal Sci J. 2013; 7 (1): 99-108.

[66] Bauer S, Halfens RJG, Lohrmann C. Knowledge and attitudes of nursing staff towards malnutrition care in nursing homes: A multicentre cross-sectional study. J Nutr Heal Aging. 2015; 19 (7): 734-740. doi: 10.1007/s12603-015-0535-7.

[67] Mowe M, Bosaeus I, Rasmussen $\mathrm{HH}$, et al. Insufficient nutritional knowledge among health care workers? Clin Nutr. 2008; 27 (2): 196-202. doi: 10.1016/j.clnu.2007.10.014.

[68] Tafese Z, Shele A. Knowledge, attitude and practice towards malnutrition among health care workers in Hawassa City, Southern Ethiopia. Glob Acad Res Journals. 2015; 1 (1): 1-8.

[69] Grammatikopoulou MG, Katsouda A, Lekka K, et al. Is continuing medical education sufficient? Assessing the clinical nutrition knowledge of medical doctors. Nutrition. 2019; 57: 69-73. doi: 10.1016/j.nut.2018.05.013.

[70] Daradkeh GA, Al Bader K, Singh R. The nutrition knowledge of primary care physicians in the state of Qatar. Pakistan J Nutr. 2012; 11 (8): 781-785.
[71] Ameh PO, Yakubu K, Miima M, Popoola O, Mohamoud G, von Pressentin KB. Lifestyle, cardiovascular risk knowledge and patient counselling among selected sub-Saharan African family physicians and trainees. African J Prim Heal Care Fam Med. 2019; 11 (1): 1-15. doi: 10.4102/phcfm.v11i1.1701.

[72] $\mathrm{Hu}$ SP, Wu MY, Liu JF. Nutrition knowledge, attitude and practice among primary care physicians in Taiwan. J Am Coll Nutr. 1997; 16 (5): 439-442. doi: 10.1080/07315724.1997.10718711.

[73] $\mathrm{Hu} \mathrm{S}, \mathrm{Wu} \mathrm{M}$, Liu J. Nutrition knowledge, attitude and practice among primary care physicians in Taiwan. J Am Coll Nutr. 1997; 16 (5): 439-442.

[74] Trakman GL, Forsyth A, Hoye R, Belski R. Developing and validating a nutrition knowledge questionnaire: Key methods and considerations. Public Health Nutr. 2017; 20 (15): 2670-2679. doi: 10.1017/S1368980017001471.

[75] Fincham JE. Response rates and responsiveness for surveys, standards, and the Journal. Am J Pharm Educ. 2008; 72 (2): 43. doi: $10.5688 /$ aj720243.

[76] Morton SMB, Bandara DK, Robinson EM, Atatoa Carr PE. In the 21 st Century, what is an acceptable response rate? Aust N Z J Public Health. 2012; 36 (2): 106-108. doi: 10.1111/j.1753-6405.2012.00854.x.

[77] Mogre V, Stevens FCJ, Aryee PA, Amalba A, Scherpbier AJJA. Why nutrition education is inadequate in the medical curriculum: A qualitative study of students' perspectives on barriers and strategies. BMC Med Educ. 2018; 18 (1): 1-11. doi: 10.1186/s12909-018-1130-5.

[78] Matsumoto M, Tanaka R, Ikemoto S. Validity and reliability of a general nutrition knowledge questionnaire for Japanese adults. J Nutr Sci Vitaminol (Tokyo). 2017; 63 (5): 298-305. doi: 10.3177/jnsv.63.298.

[79] National Research Council (US) Committee on nutrition in medical education. Nutrition Education in U.S. Medical Schools. Washington (DC); 1985.

[80] Blunt SB, Kafatos A. Clinical nutrition education of doctors and medical students: Solving the Catch 22. Adv Nutr. 2019; 10 (2): 345-350. doi: 10.1093/advances/nmy082. 